\title{
LAMB-WAVE EMBEDDED NDE WITH PIEZOELECTRIC WAFER ACTIVE SENSORS FOR STRUCTURAL HEALTH MONITORING OF THIN-WALL STRUCTURES
}

\author{
Victor Giurgiutiu \\ Mechanical Engineering Department, University of South Carolina \\ Columbia, SC 29208, victorg@sc.edu
}

\begin{abstract}
Piezoelectric wafer active sensors (PWAS) are inexpensive, non-intrusive, unobtrusive devices that can be surface-mounted on existing structures or inserted between the layers of new composite structures. The PWAS can be used in both active and passive modes. PWAS generate and detect Lamb waves and enable the development of embedded NDE concepts. This paper will present two embedded NDE concepts based on the PWAS technology and Lamb waves approach. The first concept utilizes traveling Lamb waves and could be described as embedded ultrasonics. It is shown that embedded PWAS are able to reproduce most of the conventional ultrasonic techniques, such as pitch-catch, pulse-echo, and phased array. Several experiments using Lamb waves traveling in thin-wall structures are presented.
\end{abstract}

\section{INTRODUCTION}

Embedded nondestructive evaluation (E-NDE) is an emerging technology that aims at performing NDE testing with embedded ultrasonic transducers. E-NDE will allow the use of ultrasonics methodology in automated long-term structural health monitoring (SHM). SHM of aircraft structures requires small, lightweight, inexpensive, unobtrusive, minimally invasive sensors to be embedded in the airframe with minimum weight penalty and at affordable costs [1]. Such sensors should be able to scan the structure and identify the presence of defects and incipient damage.

Current ultrasonic inspection of thin wall structures (e.g., aircraft shells, storage tanks, large pipes, etc.) is a time consuming operation that requires meticulous through-thethickness C-scans of large areas. One method to increase the efficiency of thin-wall structures inspection is to utilize guided waves (e.g., Lamb waves) [2]. Guided waves propagate along the mid-surface of thin-wall plates and shallow shells. They can travel at relatively large distances with very little amplitude loss and offer the advantage of largearea coverage with a minimum of installed sensors [3]. Guided Lamb waves have opened new opportunities for cost-effective detection of damage in aircraft structures [4]. Traditionally, guided waves have been generated by impinging the plate obliquely with a tone-burst from a relatively large ultrasonic transducer using Snell's law to achieve mode conversion. Conventional Lamb-wave transducers (wedge and comb probes) are relatively heavy and expensive. They cannot be considered for large-area deployment on an aircraft structure as part of an SHM system. Hence, a different type of sensors must be developed for SHM systems, such as the piezoelectric wafer active sensors (PWAS).

\section{PIEZOELECTRIC WAFER ACTIVE SENSORS - PWAS}

In recent years, several investigators $[5,6]$ have explored the generation of Lamb waves with piezoelectric wafer active sensors (PWAS)[7,8]. PWAS are inexpensive, non- 
intrusive, un-obtrusive, and minimally invasive devices that can be surface-mounted on existing structures, or inserted between the layers of lap joints or inside composite materials. Typical PWAS weigh around $68 \mathrm{mg}$, are $0.2 \mathrm{~mm}$ thick, and cost \$7. PWAS operated on the piezoelectric principle that couples the electrical and mechanical variables in the material (mechanical strain, $S_{i j}$, mechanical stress, $T_{k l}$, electrical field, $E_{k}$, and electrical displacement $D_{j}$ ) in the form

$$
\begin{aligned}
& S_{i j}=s_{i j k l}^{E} T_{k l}+d_{k i j} E_{k} \\
& D_{j}=d_{j k l} T_{k l}+\varepsilon_{j k}^{T} E_{k},
\end{aligned}
$$

where $s_{i j k l}^{E}$ is the mechanical compliance of the material measured at zero electric field ( $E$ $=0), \varepsilon_{j k}^{T}$ is the dielectric permittivity measured at zero mechanical stress $(T=0)$, and $d_{k i j}$ represents the piezoelectric coupling effect. For embedded NDE applications, PWAS couple their in-plane motion (which is excited through the piezoelectric effect by an oscillatory applied voltage) with the Lamb-waves particle motion on the material surface. Lamb waves can be either quasi-axial $\left(\mathrm{S}_{0}, \mathrm{~S}_{1}, \mathrm{~S}_{2}, \ldots\right)$, or quasi-flexural $\left(\mathrm{A}_{0}, \mathrm{~A}_{1}, \mathrm{~A}_{2}, \ldots\right)$. PWAS probes can act as both exciters and detectors of the elastic Lamb waves traveling in the material. For NDE, PWAS can be used as both active and passive probes.

PWAS operation is different from that of conventional ultrasonic probes:

1) PWAS are coupled to the Lamb waves through in-plane surface strains; conventional ultrasonic transducers are coupled through out-of-plane stresses that acts normal to the material surface

2) PWAS are strongly coupled with the structure and follow the structural dynamics; conventional ultrasonic probes are relatively free from the structure and follow their own dynamics

3) PWAS are non-resonant wide-band devices, while conventional ultrasonic probes are narrow-band resonant devices

The main advantage of PWAS over conventional ultrasonic probes lies in their small size, lightweight, low profile, and small cost. In spite of their small size, these novel devices are able to replicate many of the functions performed by conventional ultrasonic probes, as proven by the proof-of-concept laboratory demonstrations described further in this paper.

\section{LAMB MODE TUNED EXCITATION WITH PWAS TRANSDUCERS}

The excitation of Lamb waves with PWAS transducers can be studied by considering the excitation applied by the PWAS through a surface shear stress $\tau=\tau_{0}(x) e^{i \omega t}$. Using the space-domain Fourier transform analysis of the basic Lamb wave equations yields the strain and displacement solutions in the form

$$
\begin{aligned}
& \varepsilon_{x}(x, t)=\frac{1}{2 \pi} \frac{-i}{2 \mu} \int_{-\infty}^{\infty}\left(\frac{\tilde{\tau} N_{S}}{D_{S}}+\frac{\tilde{\tau} N_{A}}{D_{A}}\right) e^{i(\xi x-\omega t)} d \xi \\
& u_{x}(x, t)=\frac{1}{2 \pi} \frac{-i}{2 \mu} \int_{-\infty}^{\infty} \frac{1}{\xi}\left(\frac{\tilde{\tau} N_{S}}{D_{S}}+\frac{\tilde{\tau} N_{A}}{D_{A}}\right) e^{i(\xi x-\omega t)} d \xi
\end{aligned}
$$

where $\tilde{\tau}$ is the Fourier transform of $\tau_{a}(x), \quad p^{2}=\frac{\omega^{2}}{c_{L}^{2}}-\xi^{2}, \quad q^{2}=\frac{\omega^{2}}{c_{T}^{2}}-\xi^{2}$, while $c_{L}^{2}=(\lambda+2 \mu) / \rho$ and $c_{T}^{2}=\mu / \rho$ are the longitudinal (pressure) and transverse (shear) wave speeds, $\lambda$ and $\mu$ are Lamb constants, $\rho$ is the mass density, and 


$$
\begin{aligned}
& N_{S}=\xi q\left(\xi^{2}+q^{2}\right) \cos p h \cos q h, \quad D_{S}=\left(\xi^{2}-q^{2}\right)^{2} \cos p h \sin q h+4 \xi^{2} p q \sin p h \cos q h \\
& N_{A}=\xi q\left(\xi^{2}+q^{2}\right) \sin p h \sin q h, \quad D_{A}=\left(\xi^{2}-q^{2}\right)^{2} \sin p h \cos q h+4 \xi^{2} p q \cos p h \sin q h
\end{aligned}
$$

Note that $p$ and $q$ depend on $\xi$, thus increasing the problem complexity. The integral in Equation (2) is singular at the roots of $D_{S}$ and $D_{A}$. The equations $D_{S}=0$ and $D_{A}=0$ are exactly the Rayleigh-Lamb characteristic equations for symmetric and anti-symmetric motions. These equations accept the simple roots:

$$
\xi_{0}^{S}, \xi_{1}^{S}, \xi_{2}^{S}, \ldots \quad \xi_{0}^{A}, \xi_{1}^{A}, \xi_{2}^{A}, \ldots
$$

corresponding to the symmetric (S) and anti-symmetric (A) Lamb wave modes. The evaluation of the integral in Equation (2) is done by the residue theorem, using a contour consisting of a semicircle in the upper half of the complex $\xi$ plane and the real axis.

For ideal bonding between the PWAS and the plate, the shear stress in the bonding layer and the corresponding space-domain Fourier transform are

$$
\tau(x)=a \tau_{0}[\delta(x-a)-\delta(x+a)], \quad \tilde{\tau}=a \tau_{0}[-2 i \sin \xi a]
$$

Hence, the strain-wave solution becomes

$$
\varepsilon_{x}(x, t)=-i \frac{a \tau_{0}}{\mu} \sum_{\xi^{S}} \sin \xi^{S} a \frac{N_{S}\left(\xi^{S}\right)}{D_{S}^{\prime}\left(\xi^{S}\right)} e^{i\left(\xi^{S} x-\omega t\right)}-i \frac{a \tau_{0}}{\mu} \sum_{\xi^{A}} \sin \xi^{A} a \frac{N_{A}\left(\xi^{A}\right)}{D_{A}^{\prime}\left(\xi^{A}\right)} e^{i\left(\xi^{A} x-\omega t\right)}
$$

Similarly, the displacement wave solution is obtained as

$$
u_{x}(x, t)=-i \frac{a \tau_{0}}{\mu} \sum_{\xi^{S}} \frac{\sin \xi^{S} a}{\xi^{S}} \frac{N_{S}\left(\xi^{S}\right)}{D_{S}^{\prime}\left(\xi^{S}\right)} e^{i\left(\xi^{S} x-\omega t\right)}-i \frac{a \tau_{0}}{\mu} \sum_{\xi^{A}} \frac{\sin \xi^{A} a}{\xi^{A}} \frac{N_{A}\left(\xi^{A}\right)}{D_{A}^{\prime}\left(\xi^{A}\right)} e^{i\left(\xi^{A} x-\omega t\right)}
$$

A plot of these equations up to $1000 \mathrm{kHz}$ is presented in Figure 1. Equations (6) and (7) contains the $\sin \xi a$ behavior that displays maxima when the PWAS length $l_{a}=2 a$ equals an odd multiple of the half wavelength, and minima when it equals an even multiple of the half wavelength. A complex pattern of such maxima and minima emerges, since several Lamb modes, each with its own different wavelength, coexist at the same time. Figure 1a shows that, at $300 \mathrm{kHz}$, the amplitude of the $\mathrm{A}_{0}$ mode goes through zero, while that of the $\mathrm{S}_{0}$ is close to its peak. This represents an excitation "sweet spot" for $\mathrm{S}_{0}$ Lamb waves. Experimental results confirming this prediction are presented in Figure $1 b$.

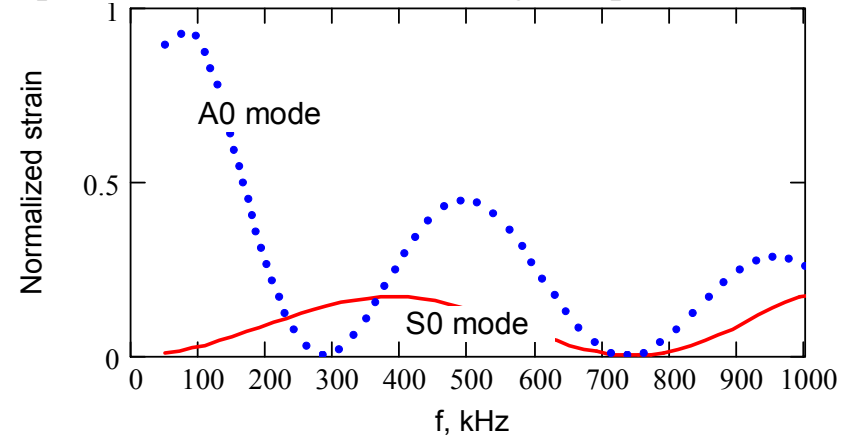

(a)

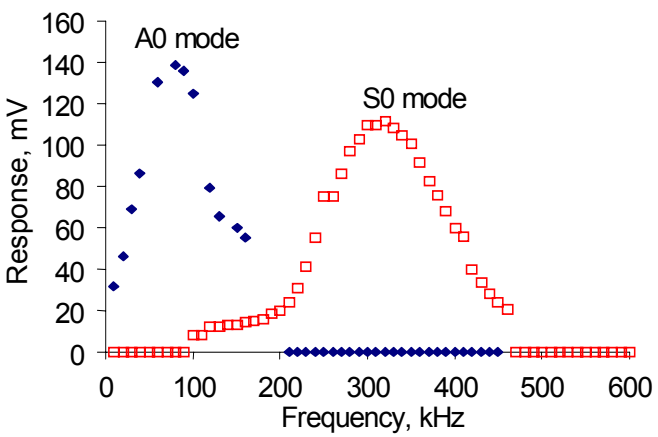

(b)

Figure 1 (a) Strain Lamb wave amplitudes predicted by the Lamb wave PWAS excitation tuning ( $\mathrm{S}_{0}=$ continuous line; $A_{0}=$ dotted line); (b) excitation sweet spot observed experimentally at $300 \mathrm{kHz}$ in a $1.6-\mathrm{mm}$ aluminum plate under a 7-mm PWAS excitation

This proves that frequencies can be found for which the response is dominated by certain modes. This is wavelength-based mode tuning. Another factor that must be considered in Lamb wave tuning under PWAS excitation is the mode amplitude at the plate surface where the PWAS is located. This factor is contained in the values taken for each mode by the function $N / D^{\prime}$. It is conceivable that, at a given frequency, some higher modes may 
have less surface amplitudes, while other may have larger surface amplitudes. Thus, two important factors for the design of PWAS-based Lamb-wave embedded NDE for structural health monitoring have been identified:

a) The variation of $|\sin \xi a|$ with frequency for each Lamb wave mode

b) The variation of the surface strain with frequency for each Lamb wave mode Equations (6) and (7) can be viewed as a superposition of waveguide Lamb modes. In this case, the contribution of each mode can be expressed in the general form

$$
A_{\xi}(x, t)=G \cdot E_{\xi} \cdot F \cdot e^{i(\xi x-\omega t)}
$$

where $G$ is a numerical factor representing the PWAS transducer power transmitted to the structure, $E_{\xi}$ is the excitability function of mode $\xi$, and $F$ is the Fourier transform of the excitation.

\section{CRACK DETECTION WITH PWAS}

Wave propagation experiments were conducted on an aircraft panel to illustrate crack detection through the pulse-echo method. The panel had a typical aircraft construction, featuring a vertical splice joint and horizontal stiffeners. Figure 2 shows three photographs of PWAS installation on increasingly more complex regions of the panel. Adjacent to the photographs are the PWAS signals. All the experiments used only one PWAS, operated in pulse-echo mode. The PWAS was placed in the same relative location, i.e., at $200 \mathrm{~mm}$ to the right of the vertical row of rivets. The photo on the first row of Figure 2 shows the situation with the lowest complexity, in which only the vertical splice joint and the vertical row of rivets is present in the far left. The signal to the right of this photograph shows the initial bang (centered at around 5.3 micro-sec) and multiple reflections from the panel edges and the splice joint. The echoes start to arrive at approximately $60 \mu \mathrm{s}$. The photo on the second row of Figure 2 shows the vertical row of rivets in the far left and, in addition, a horizontal double row of rivets stretching towards the PWAS. The signal to the right shows that, in addition to the multiple echoes from the panel edges and the splice, the PWAS also receives backscatter echoes from the rivets located at the beginning of the horizontal row. These backscatter echoes are visible at around $42 \mu \mathrm{s}$. The photo on the third row in Figure 2 shows a region of the panel similar to that presented in the previous row, but having an addition feature: a simulated crack (12.7-mm EDM hairline slit) emanating from the first rivet hole in the top horizontal row. The signal at the right of this photo shows features similar to those of the previous signal, but somehow stronger at the $42 \mu$ s position. The features at $42 \mu \mathrm{s}$ correspond to the superposed reflections from the rivets and from the crack. The detection of the crack seems particularly difficult because the echoes from the crack and from the rivets are superposed. This difficulty was resolved through the differential signal method, i.e., subtracting the signal recorded on the undamaged structure (second row) from the signal on the damaged structure (third row). The result is presented in the last row of Figure 2, which shows a "loud and clear" echo that is due entirely to the crack. This echo, is centered at $42 \mu$ s, i.e., TOF $=37 \mu$ s which correlates very well with a $200-\mathrm{mm}$ roundtrip travel at $5.4 \mathrm{~km} / \mathrm{s}$ from the PWAS to the crack. The cleanness of the crack-detection feature and the quietness of the signal ahead of the crack-detection feature are very good. Thus, we concluded that PWAS are capable of clean and un-ambiguous detection of structural cracks. 

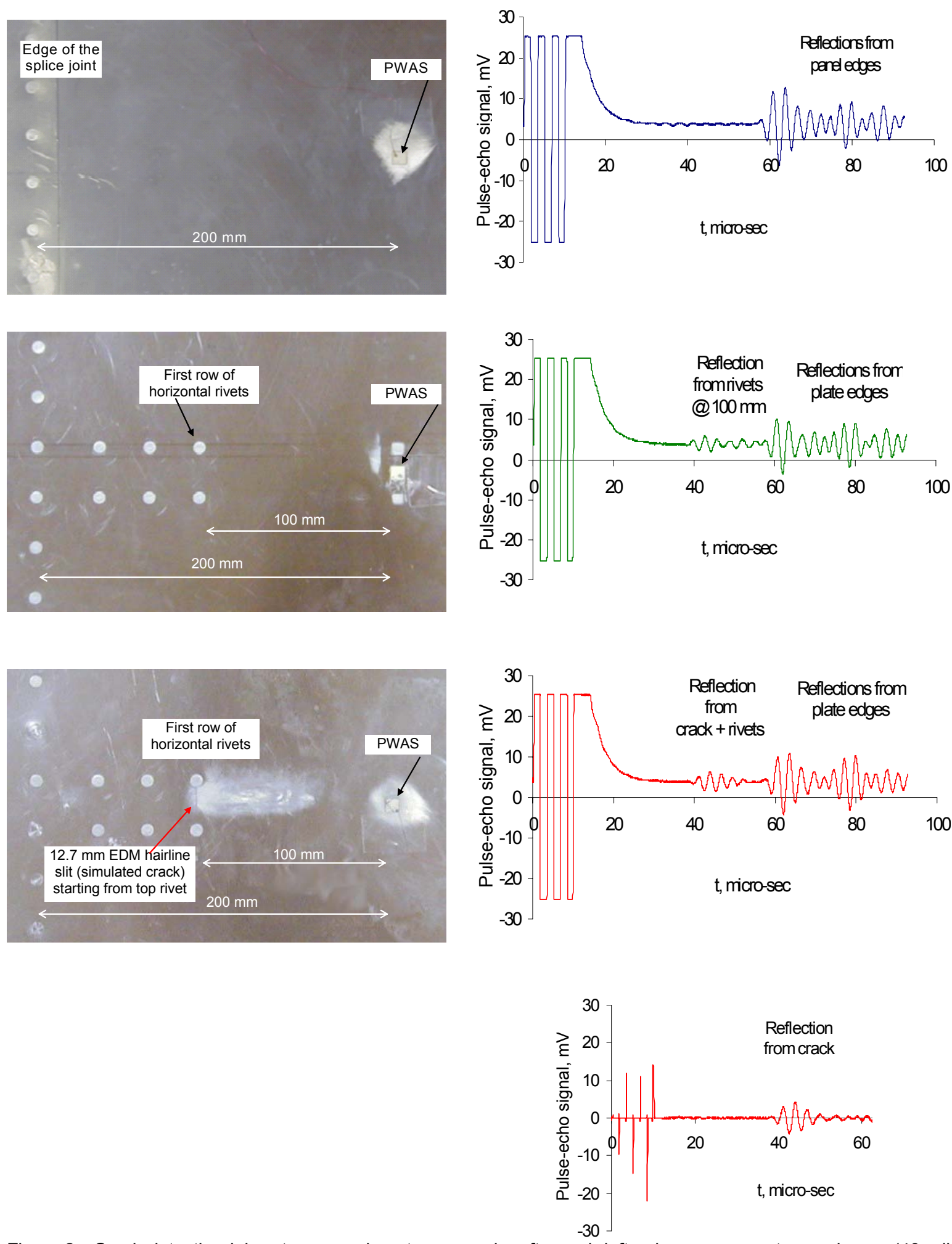

Figure 2 Crack-detection laboratory experiments on an aircraft panel: left column represents specimens (40-mil 2025 T3) with increasing complexity. Right column represents the pulse-echo signals. Fourth cell in the right column shows the crack detection through the differential signal method. 


\section{PWAS PHASED ARRAYS}

The advantages of phased array transducers for ultrasonic testing are multiple [9]. In our research [10], we have developed a phased array concept for thin wall structures (e.g., aircraft shells, storage tanks, large pipes, etc.) that uses PWAS-generated Lamb waves to cover a large surface area through beam steering from a central location. We called this concept embedded ultrasonics structural radar (EUSR). Simple proof-of-concept experiments were constructed to illustrate this method. A PWAS array made up of nine identical 7-mm sq. PWAS elements at uniform 9-mm pitch was constructed. The PWAS phased array was placed at the center of a 4 -ft square thin aluminum plate. The wave pattern generated by the phased array is the result of the superposition in virtual time of the waves generated by each individual element.

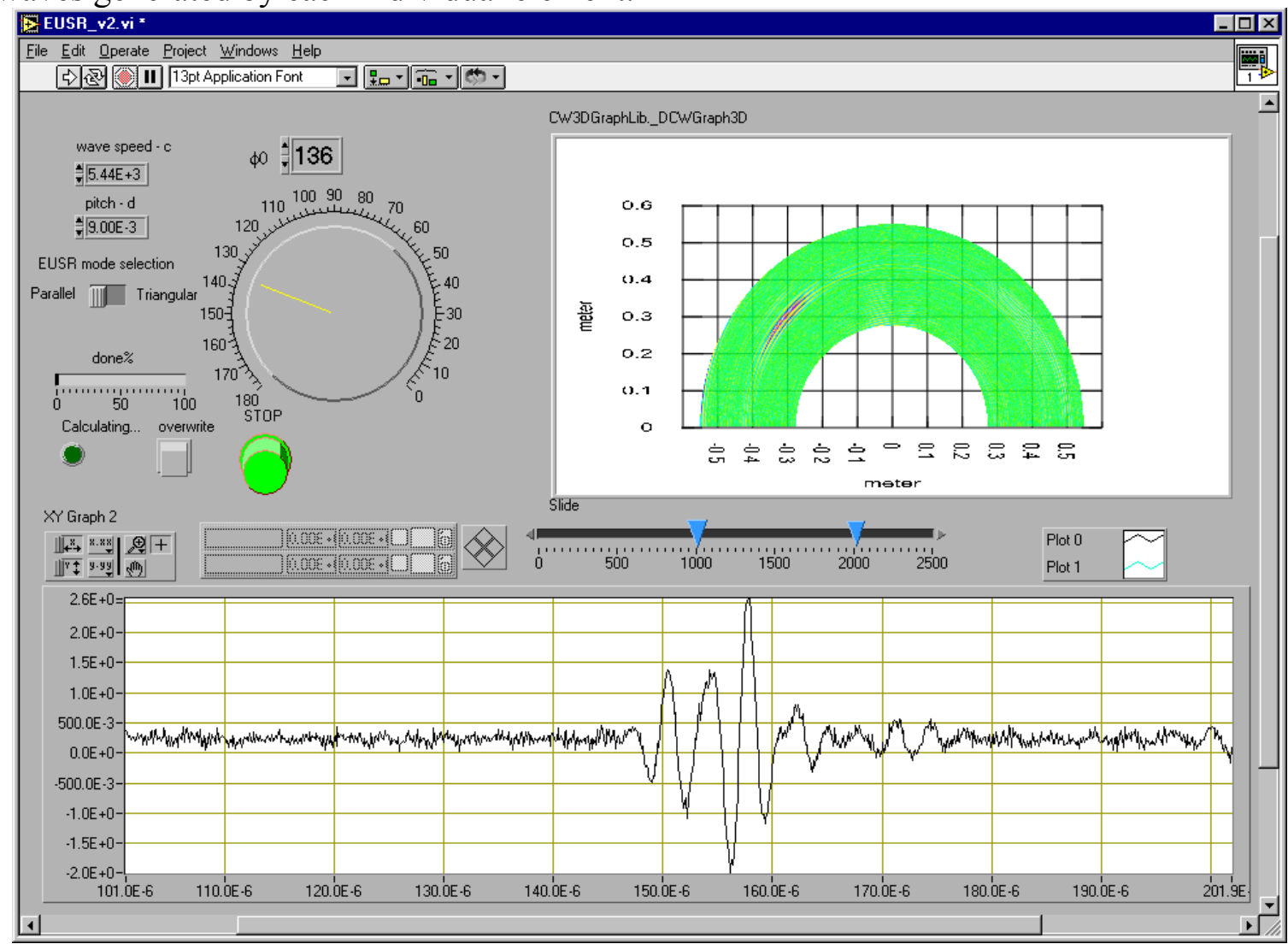

Figure 3 - Proof-of-concept EUSR experiment: (a) thin plate specimen 9-element PWAS array and 19-mm offside crack; (b) Graphical user interface (EUSR-GUI) front panel. The angle sweep is performed automatically to produce the structure/defect imaging picture on the right. Manual sweep of the beam angle can be also performed with the turn knob; the signal reconstructed at the particular beam angle (here, $\phi_{0}=136$ deg) is shown in the lower picture.

In conventional phased array transducers, the ultrasonic wave front can be focused or steered in a specific direction by sequentially firing the individual elements of an array transducer at slightly different times. This approach requires a large number of excitation channels working in parallel (one for each element of the phased array). This would impose a large hardware burden on the SHM system. To reduce the hardware burden, we took a novel approach. We applied a technique in which only one PWAS in the array is excited at a given time, while data is being collected on all the PWAS in the array. Thus the hardware requirements were reduced to just one excitation channel. The excitation was then switched among all the PWAS in the array in a round-robin manner. Eventually, an array of $N^{2}$ primitive signals was generated and collected (here, $N=9$ ). An algorithm for creating a virtual beam-steering/focusing effect (called the EUSR algorithm) was applied to the array 
of $N^{2}$ primitive signals. Once the beam steering and focusing were established, the crack detection was done with the pulse-echo method. Two proof-of-concept experiments were conducted: (i) detection of a broadside crack placed at $305 \mathrm{~mm}$ from the array in the $90 \mathrm{deg}$ direction; and (ii) detection of an offside crack placed at $409 \mathrm{~mm}$ from the array in the 136 deg direction. Both cracks were $19 \mathrm{~mm}$ long and oriented parallel to the array. Of these two experiments, the latter was more challenging because the ultrasonic beam is not reflected back to the array but rather deflected away from the array. The only "echo" received from the offside crack is the backscatter of the diffraction signal generated at the crack tips. Figure 3 presents the front panel of the embedded ultrasonic structural radar graphical user interface (EUSR-GUI) when detecting the offside crack. The structural defect is imaged in the pane presented in the right upper corner. This imaging is done by automatic sweep. Manual sweep can be also performed using the turn knob located in the upper left corner. The reconstructed signal shown in the lower pane is an A scan corresponding to the knob position. The A scan shown in Figure 3 is the reconstructed signal corresponding to a beam angle $\phi_{0}=136 \mathrm{deg}$, which is in the direction of the crack. thus, we have proved that widearea inspection is possible with a PWAS array generating a sweeping beam of ultrasonic Lamb waves that can cover the whole plate.

\section{SUMMARY AND CONCLUSIONS}

This paper has presented a novel NDE concept - embedded NDE with piezoelectric wafer active sensors (PWAS). This concept can be one of the enabling technologies for the implementation of structural health-monitoring (SHM) of thin-wall structures. The embedded NDE concept opens new opportunities for performing in-situ damage detection and structural health monitoring of a multitude of thin-wall structures such as aircraft, spacecraft, missiles, pressure vessels, oil tanks, pipelines, etc. PWAS are inexpensive, nonintrusive, unobtrusive, and minimally invasive devices that can be surface-mounted on existing structures, inserted between the layers of lap joints, and even inside composite materials. PWAS can be structurally embedded as both individual transducers and as phased arrays. PWAS can be placed inside closed cavities during structural fabrication or overhaul and then left in place for the life of the structure. Since the structure is closed up after PWAS are placed inside it, the PWAS become embedded into the structure. Such situations may arise when PWAS are placed inside aircraft wing structures, aircraft fuselages, in nuclear power plant piping, or in other closed-up structures.

PWAS operation is different from that of conventional ultrasonic probes:

4) PWAS are coupled to the Lamb waves through in-plane surface strains; conventional ultrasonic transducers are coupled through out-of-plane stresses that act normal to the material surface

5) PWAS are strongly coupled with the structure and follow the structural dynamics; conventional ultrasonic probes are relatively free from the structure and follow their own dynamics

6) PWAS are non-resonant wide-band devices; conventional ultrasonic transducers are narrow-band resonant devices

The main advantage of PWAS over conventional ultrasonic probes lies in their small size, lightweight, low profile, and small cost. In spite of their small size, these novel devices are able to replicate many of the functions performed by conventional ultrasonic probes, as proven by the proof-of-concept laboratory presented in this paper.

This paper has shown theoretically and experimentally that PWAS transducers can selectively excite various Lamb wave guided modes. The analysis was performed with the space-domain Fourier transform. An excitation "sweet spot" for the $\mathrm{S}_{0}$ Lamb waveguide mode was predicted at around $300 \mathrm{kHz}$. This prediction was experimentally verified with 
good accuracy. Systematically conducted experiments verified that "loud and clear" ultrasonic Lamb waves can be successfully generated with the small, inexpensive, and unobtrusive PWAS transducers. This opened the way for applying the pulse-echo crackdetection method which was demonstrated on a realistic aircraft panel. The differential signal method was used to detect the presence of a crack in a complicated structural setup. The arrangement of PWAS in phased arrays was also explored. The embedded ultrasonics structural radar (EUSR) concept was presented. This concept utilizes a virtual beam forming and beam focusing algorithm based on an array of $N^{2}$ primitive signals collected in a round-robin fashion. The EUSR concept requires an order of magnitude less instrumentation than the conventional phased-array devices. The sweeping of a large structural area from a central location and the successful detection of broadside and offside cracks was demonstrated on a $1.2-\mathrm{m}$ square thin aluminum plate.

Though the potential capabilities of the PWAS-based embedded NDE have been demonstrated, this emerging technology still requires a sustained R\&D effort in order to achieve its full development potential and be applied to full-scale structures.

\section{ACKNOWLEDGMENTS}

Support from the US Air Force Research Laboratory through contract \#03-S470-033-C1 of F33615-01-D-5801, and from the Department of Energy through the Sandia National Laboratories contract doc. \# BF 0133 are thankfully acknowledged.

\section{REFERENCES}

1. Kropas-Hughes, C.V.; Perez, I.; Winfree; W. P.; Motzer, W. P.; Thompson, R. B. (2002) "Vision of Future Directions of NDE Research" in Review of Quantitative Nondestructive Evaluation Vol. 21, ed. by D. O. Thompson and D. E. Chimenti, American Institute of Physics, Vol. 615, 2002, pp. 2042-2051

2. Rose, J. L. (2002) "A Baseline and Vision of Ultrasonic Guided Wave Inspection Potential", ASME Journal of Pressure Vessel Technology - Special Issue on Nondestructive Characterization of Structural Materials, Vol. 124, No. 3, August 2002, pp. 273-282

3. Viktorov, I. A. (1967) Rayleigh and Lamb Waves, Plenum Press, New York, 1967

4. Dalton, R. P.; Cawley, P.; Lowe, M. J. S. (2001) "The Potential of Guided Waves for Monitoring Large Areas of Metallic Aircraft Structure", Journal of Nondestructive Evaluation, Vol. 20, pp. 29-46, 2001

5. Keilers, C. H.; Chang, F.-K. (1995) "Identifying Delamination in Composite Beam using Built-in Piezoelectrics", Journal of Intelligent Material Systems and Structures, Vol. 6, 1995, pp. 647672

6. Chang, F.-K. (1995) "Built-In Damage Diagnostics for Composite Structures", in Proceedings of the $10^{\text {th }}$ International Conference on Composite Structures (ICCM-10), Vol. 5, Whistler, B. C., Canada, August 14-18, 1995, pp.283-289

7. Giurgiutiu, V.; Zagrai, A. (2000) "Characterization of Piezoelectric Wafer Active Sensors", Journal of Intelligent Material Systems and Structures, Technomic Pub., USA, Vol. 11, No. 12, December 2000, pp. 959-976

8. Giurgiutiu, V.; Zagrai, A. N.; Bao, J. (2002) "Embedded Active Sensors for In-Situ Structural Health Monitoring of Thin-Wall Structures", ASME Journal of Pressure Vessel Technology, Vol. 124, No. 3, August 2002, pp. 293-302

9. Lines D.; Dickson K. (1999) "Optimization of High-Frequency Array Technology for Lap-Joint Inspection"; Proceedings of the $3^{\text {rd }}$ Joint Conference on Aging Aircraft, 1999, www.galaxyscientific.com/agingaircraft/index $2 . \mathrm{htm}$

10.Giurgiutiu, V.; Bao, J. (2002) "Embedded Ultrasonic Structural Radar for the Nondestructive Evaluation of Thin-Wall Structures" Proceedings of the 2002 ASME International Mechanical Engineering Congress, November 17-22, 2002, New Orleans, LA, paper \# IMECE2002-39017 